\title{
MIMAC: A micro-tpc matrix for directional detection of dark matter
}

\author{
D. Santos*, J. Billard, G. Bosson, O. Bourrion, C. Grignon, O. Guillaudin, F. Mayet, \\ J.P. Richer \\ LPSC, Universite Joseph Fourier Grenoble 1, CNRS/IN2P3, Institut Polytechnique de Grenoble \\ E-mail: Daniel.Santos@lpsc.in2p3.fr \\ E. Ferrer, I. Giomataris, F.J. Iguaz, J.P. Mols \\ IRFU,CEA Saclay, 91191 Gif-sur-Yvette cedex
}

\section{A. Allaoua, C. Golabek, L. Lebreton}

LMDN, IRSN Cadarache, 13115 Saint-Paul-Lez-Durance

\begin{abstract}
Directional detection of non-baryonic Dark Matter is a promising search strategy for discriminating WIMP events from background. However, this strategy requires both a precise measurement of the energy down to a few $\mathrm{keV}$ and $3 \mathrm{D}$ reconstruction of tracks down to a few $\mathrm{mm}$. To achieve this goal, the MIMAC project has been developed. It is based on a gaseous micro-TPC matrix, filled with ${ }^{3} \mathrm{He}, \mathrm{CF}_{4}$ and/or $\mathrm{C}_{4} \mathrm{H}_{10}$. The first results on low energy nuclear recoils $\left({ }^{1} \mathrm{H}\right.$ and $\left.{ }^{19} \mathrm{~F}\right)$ obtained with a low mono-energetic neutron field are presented. The discovery potential of this search strategy is discussed and illustrated by a realistic case accessible to MIMAC.
\end{abstract}

Identification of Dark Matter 2010-IDM2010

July 26-30, 2010

Montpellier France

\footnotetext{
*Speaker.
} 


\section{Introduction}

Directional detection of Dark Matter is based on the fact that the solar system moves with respect to the center of our galaxy with a mean velocity of roughly $220 \mathrm{~km} / \mathrm{s}$ [1]. Taking into account the hypothesis of the existence of a galactic halo of DM formed by WIMPs (Weakly Interacting Particles) with a negligible rotation velocity, we can expect a privileged direction for the nuclear recoils in our detector, coming out from elastic collision with those WIMPs.

The MIMAC (MIcro-tpc MAtrix of Chambers) detector project tries to get these elusive events by a double detection: ionization and track, at low gas pressure with low mass target nuclei $(\mathrm{H}, 19 \mathrm{~F}$, $3 \mathrm{He}$ ). In order to have a significant cross section we explore the axial, spin dependant, interaction on odd nuclei. The very weak correlation between the neutralino-nucleon scalar cross section and the axial one, as it was shown in [2], makes this research, at the same time, complementary to the massive target experiments.

\section{MIMAC prototype}

The MIMAC prototype consists of one of the chamber of the matrix allowing to show the ionization and track measurement performances needed to achieve the directional detection strategy. The primary electron-ion pairs produced by a nuclear recoil in one chamber of the matrix are detected by driving the electrons to the grid of a bulk micromegas[3] and producing the avalanche in a very thin gap (128 or $256 \mu \mathrm{m})$.
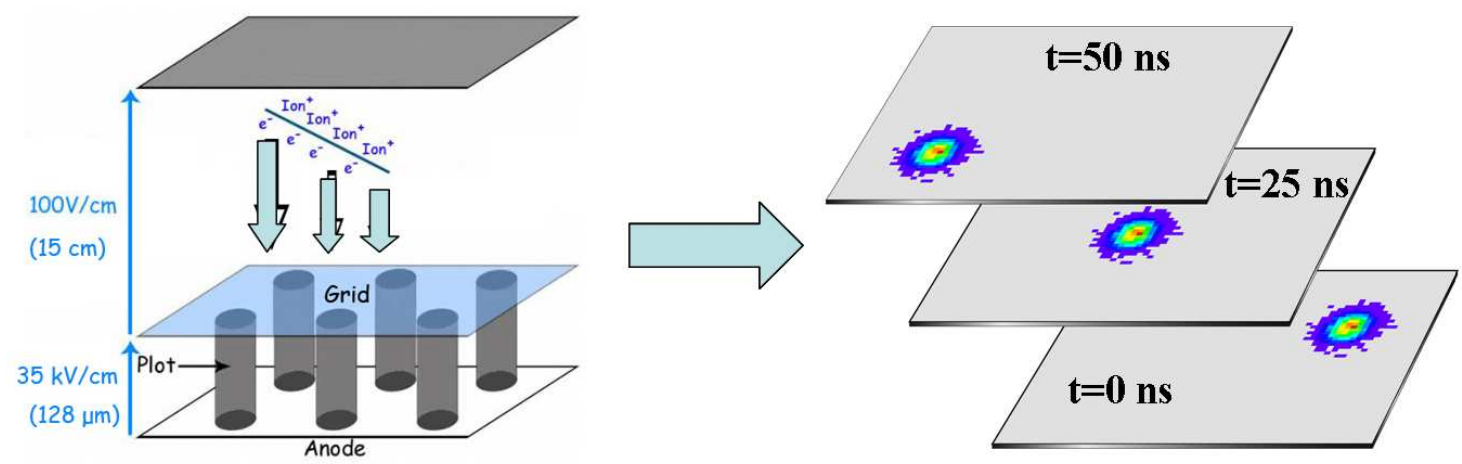

Figure 1: Track reconstruction in MIMAC. The anode is read-out every $25 \mathrm{~ns}$ and the 3D track is recontructed, knowing the drift velocity of primary electrons, from the consecutive number of images, defining the event, from the anode.

As pictured on figure 1, the electrons move towards the grid in the drift space and are projected on the anode thus allowing to get information on $\mathrm{X}$ and $\mathrm{Y}$ coordinates. To access the $\mathrm{X}$ and $\mathrm{Y}$ dimensions with a $100 \mu \mathrm{m}$ spatial resolution, a bulk micromegas with a 4 by $4 \mathrm{~cm}$ active area, segmented in pixels with a pitch of $350 \mu \mathrm{m}$ is used as $2 \mathrm{D}$ readout. In order to reconstruct the third dimension $\mathrm{Z}$ of the recoil, the LPSC developed a self-triggered electronics able to perform the anode sampling at a frequency of $40 \mathrm{MHz}$. This includes a dedicated 16 channels ASIC [7] associated to a DAQ [8]. 
In order to get the total recoil energy we need to know the ionization quenching factor (IQF) of the nuclear recoil in the gas used. We have developped at the LPSC a dedicated experimental facility to measure such IQF. A precise assessment of the available ionization energy has been performed by Santos et al. [4] in $\mathrm{He}+5 \% \mathrm{C}_{4} \mathrm{H}_{10}$ mixture within the dark matter energy range (between 1 and $50 \mathrm{keV}$ ) by a measurement of the IQF. For a given energy, an electron track in a low pressure micro-TPC is an order of magnitude longer than a recoil one. It opens the possibility to discriminate electrons from nuclei recoils by using both energy and track length informations, as it was shown in [5]. The 3D tracks are obtained from consecutive read-outs of the anode, every $25 \mathrm{~ns}$, defining the event. To get the length and the orientation of the track, an independent mesurement of the drift velocity is needed. Preliminary measurements, with different gases in the MIMAC prototype, of the drift velocity have been performed and they fit well with Magboltz simulations [6].

\section{First experimental results}

The first result concerning the ability to detect tracks with the prototype was performed with a ${ }^{55} \mathrm{Fe} \mathrm{X}$-ray source in order to reconstruct the $5.9 \mathrm{keV}$ electron tracks produced by photoelectric effect in the active volume.
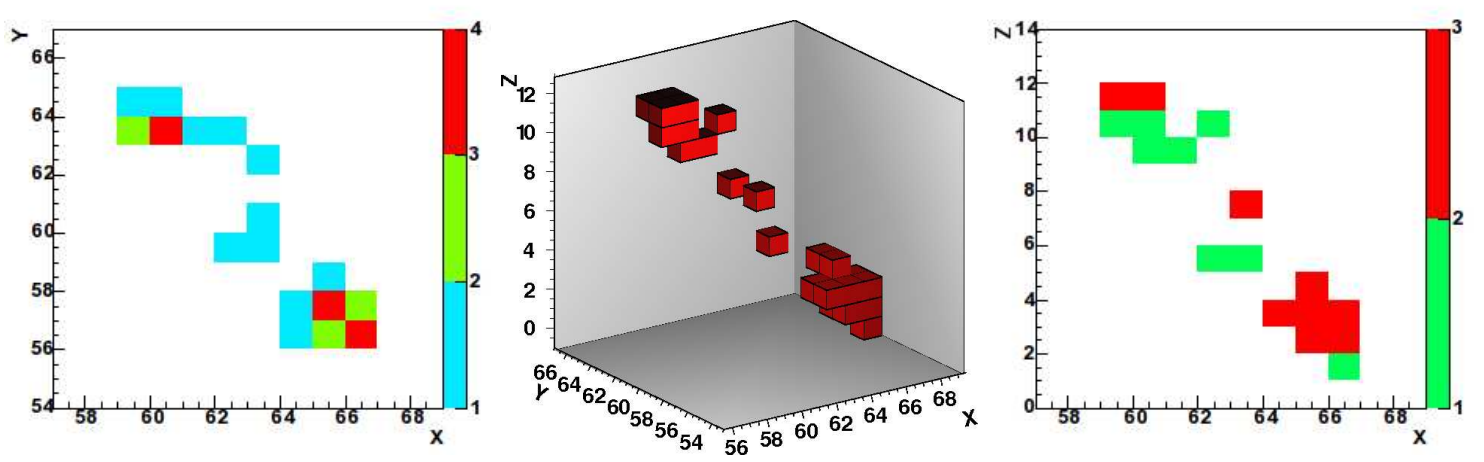

Figure 2: A $5.9 \mathrm{keV}$ electron track in 350 mbar $95 \%{ }^{4} \mathrm{He}+\mathrm{C}_{4} \mathrm{H}_{10}$. The left panel represents the 2D projection of the recoil seen by the anode, the center panel represents a $3 \mathrm{D}$ view of the track after using the reconstruction algorithm and the right panel represents a projection of the $3 \mathrm{D}$ track on the $\mathrm{XZ}$ plane

Figure 2 presents a typical electron track seen by the anode (X,Y) (left panel), its projection on the $\mathrm{XZ}$ plane (right panel) and reconstructed in 3D (center panel). This result shows the MIMAC capability to reconstruct the track of low energy electrons which are the typical background in dark matter experiments.

On fig 3 (left panel), a 3D track reconstruction is achieved for high energy (5.5 MeV) alpha particles issued from the natural radioactivity $\left({ }^{222} \mathrm{Rn}\right)$ present in the chamber. However, the final validation concerning the possibility for MIMAC to get directional detection had to be done with neutrons giving nuclear recoils in the range of a few $\mathrm{keV}$. In order to have monoenergetic neutron fields, in the range of a few tens of $\mathrm{keV}$, we perform an experiment at the AMANDE facility (IRSNCadarache) allowing to select the energy of the neutrons by the angle with respect to a proton beam producing a neutron resonance on a LiF target. 

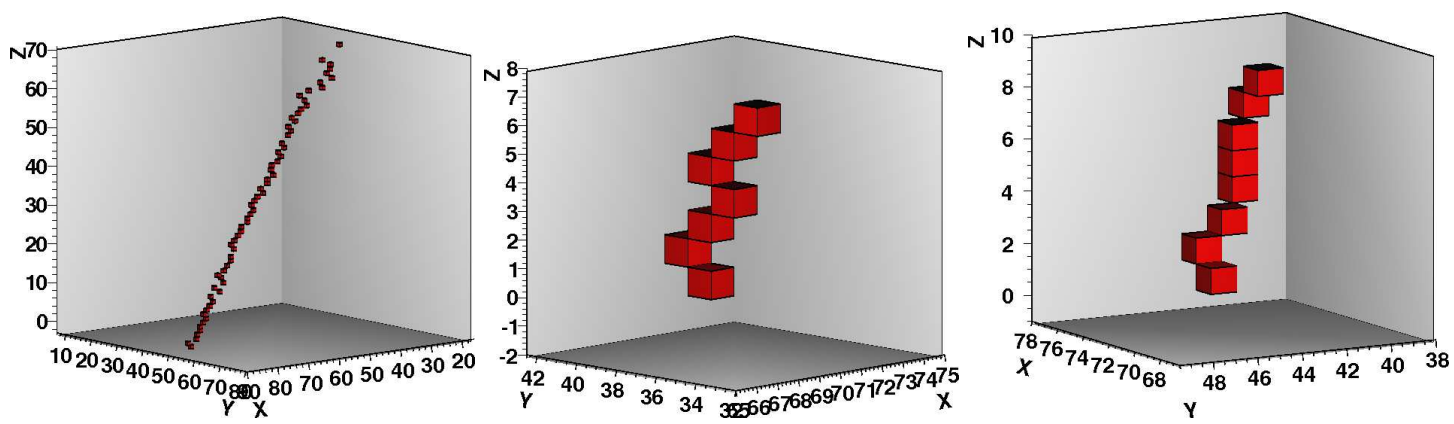

Figure 3: From left to right: recoil of $5.5 \mathrm{MeV} \mathrm{He}$ nuclei in 350 mbar ${ }^{4} \mathrm{He}+5 \% \mathrm{C}_{4} \mathrm{H}_{10}, 8 \mathrm{keV}$ hydrogen nucleus in 350 mbar $^{4} \mathrm{He}+5 \% \mathrm{C}_{4} \mathrm{H}_{10}$ and fluorine nucleus leaving $50 \mathrm{keV}$ in ionization in $55 \mathrm{mbar} 70 \% \mathrm{CF}_{4}$ $+30 \% \mathrm{CHF}_{3}$

On fig 3 (center and right panel), 3D tracks of nuclear recoils following elastic scattering of mono-energetic neutrons are represented. On the center panel, a $8 \mathrm{keV}$ proton recoil leaving a track of $2.4 \mathrm{~mm}$ long in $350 \mathrm{mbar}{ }^{4} \mathrm{He}+5 \% \mathrm{C}_{4} \mathrm{H}_{10}$ is represented. This event is a typical kind of signal that MIMAC will expect for dark matter search. The right panel presents a $50 \mathrm{keV}$ (in ionization) fluorine recoil of $3 \mathrm{~mm}$ long obtained in a 55 mbar mixture of $70 \% \mathrm{CF}_{4}+30 \% \mathrm{CHF}_{3}$. In addition, the electron-recoil discrimination, a very important point for dark matter detection, showing the ability to separate gamma background from nuclear recoils, has been presented in pure Isobutane or ${ }^{4} \mathrm{He}+5 \% \mathrm{C}_{4} \mathrm{H}_{10}$ mixture in ref. [5].

\section{Directional detection of Dark Matter and its discovery potential}

Directional detection of non-baryonic Dark Matter is a promising search strategy for discriminating WIMP events from background offering a complementarity to massive detectors $[9,10]$. The directionality gives a solid and unambiguous signature of the correlation between the galactic halo and a signal in our detector. This is achieved by searching for a correlation of the WIMP signal with the solar motion around the galactic center, observed as a direction dependence of the WIMP flux coming roughly from the direction of the Cygnus constellation [1]. This is generally referred to as directional detection of Dark Matter and several projects are being developed for this goal $[11,12,13,14,15]$.

The main asset of directional detection is the fact that the WIMP angular distribution is pointing towards the Cygnus constellation while the background one is isotropic (fig 4). The right panel of figure 4 presents a typical recoil distribution observed by a directional detector : 100 WIMP-induced events and 100 background events generated isotropically. For an elastic axial cross-section on nucleon $\sigma_{\mathrm{n}}=1.5 \times 10^{-3} \mathrm{pb}$ and a $100 \mathrm{GeV} . \mathrm{c}^{-2}$ WIMP mass, this corresponds to an exposure of $\sim 7 \times 10^{3} \mathrm{~kg}$.day in ${ }^{3} \mathrm{He}$ and $\sim 1.6 \times 10^{3} \mathrm{~kg}$.day in $\mathrm{CF}_{4}$, on their equivalent energy ranges as discussed in ref. [16]. Low resolution maps are used in this case $\left(N_{\text {pixels }}=768\right)$ which is sufficient for the low angular resolution, $\sim 15^{\circ}$ (FWHM), expected for this type of detector. In this case, 3D read-out and sense recognition are considered, while background rejection is based on electron/recoil discrimination by track length and energy selection [5]. It is not straightforward 

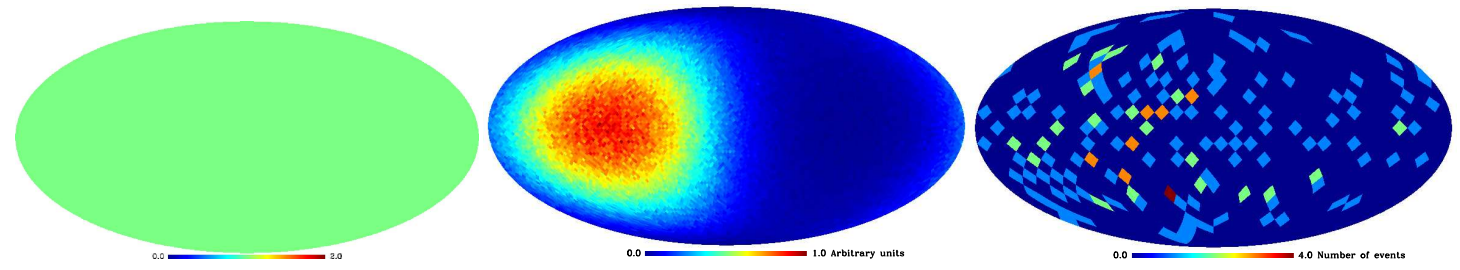

Figure 4: From left to right : isotropic background distribution, WIMP-induced recoil distribution in the case of an isothermal spherical halo and a typical simulated measurement : 100 WIMP-induced recoils and 100 background events with a low angular resolution. Recoils maps are produced for a ${ }^{19} \mathrm{~F}$ target, a 100 GeV.c ${ }^{-2}$ WIMP and considering recoil energies in the range $5 \mathrm{keV} \leq E_{R} \leq 50 \mathrm{keV}$. Figures from [16].

to conclude from the recoil map of figure 4 (right) that it does contain a fraction of WIMP events pointing towards the direction of the solar motion.

To extract information from this example of a measured map, a likelihood analysis has been developed. The likelihood value is estimated using a binned map of the overall sky with Poissonian statistics, as shown in Billard et al. [16]. This is a four parameter likelihood analysis with $m_{\chi}$, $\lambda=S /(B+S)$ the WIMP fraction ( $B$ is the background spatial distribution taken as isotropic and $S$ is the WIMP-induced recoil distribution) and the coordinates $(\ell, b)$ referring to the maximum of the WIMP event angular distribution.
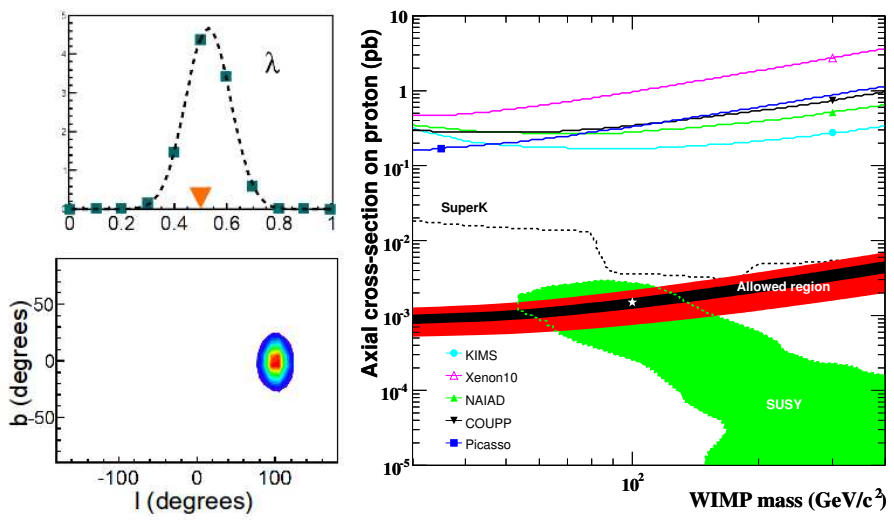

Figure 5: On the left, marginalized probability density functions of $\lambda$ (top), $\ell, b$ (bottom) after the likelihood analysis of the simulated recoil map of fig 4 right. On the right, allowed regions obtained with the example map shown on figure 4 presented in the plane of the spin dependent cross-section on proton ( $\mathrm{pb}$ ) as a function of the WIMP mass $\left(\mathrm{GeV} / \mathrm{c}^{2}\right)$. Input value for the simulation is shown with a star. Figures from [16].

The result of this map-based likelihood method is that the main recoil direction is recovered and it is pointing towards $\left(\ell=95^{\circ} \pm 10^{\circ}, b=-6^{\circ} \pm 10^{\circ}\right)$ at $68 \% \mathrm{CL}$, corresponding to a nonambiguous detection of particles from the galactic halo. This is indeed the discovery proof of this detection strategy (left panel of fig. 5) [16]. Furthermore, the method allows to constrain the WIMP fraction in the observed recoil map leading to a constraint in the $\left(\sigma_{n}, m_{\chi}\right)$ plane (right panel of fig. 5). As emphasized in ref. [16], a directional detector could allow for a high significance discovery 
of galactic Dark Matter even with a sizeable background contamination. For very low exposures, competitive exclusion limits may also be imposed [17].

\section{Conclusions}

Directional detection is a promising search strategy to discover galactic dark matter. The MIMAC detector provides the energy of a recoiling nucleus and the reconstruction of its 3D track. The first 3D tracks observed with the MIMAC prototype were shown: $5.9 \mathrm{keV}$ electrons (typical background) and low energy proton and fluorine recoils (typical signal). The next step will be to build a demonstrator of $1 \mathrm{~m}^{3}$ to show that the large micro-tpc matrix for directional detection of dark matter search is accessible.

\section{Acknowledgements}

The MIMAC collaboration acknowledges the ANR-07-BLANC-0255-03 funding.

\section{References}

[1] D. N. Spergel, Phys. Rev. D 37 (1988) 1353.

[2] E. Moulin, F. Mayet et D. Santos. Phys. Lett. B 614 (2005) 143-154.

[3] I. Giomataris et al., Nucl. Instrum. Methods A 560 (2006) 405.

[4] D. Santos et al., arXiv:0810.1137.

[5] C. Grignon et al., JINST 4 (2009) P11003, arXiv:0909.0654.

[6] S.F. Biagi, Nucl. Instrum. and Meth. A421 (1999) 234-240.

[7] J.P. Richer et al., Nucl. Instrum. Methods A 620 (2010) 470.

[8] O. Bourrion et al.,Nucl. Instrum. and Meth. A662(2010) 207.

[9] E. Aprile et al., arXiv:1005.0380.

[10] E. Armengaud et al., Phys. Lett. B 687 (2010) 294-298.

[11] D. Santos et al., J. Phys. Conf. Ser. 65 (2007) 012012.

[12] G. J. Alner et al., Nucl. Instr. Meth. A 555 (2005) 173.

[13] S. Ahlen et al., arXiv:1006.2928.

[14] K. Miuchi et al., Phys. Lett. B 654 (2007) 58.

[15] S. Ahlen et al., Int. J. Mod. Phys. A 25 (2010) 1.

[16] J. Billard et al., Phys. Lett. B 691 (2010) 156-162.

[17] J. Billard et al., Phys. Rev. D 82 (2010) 55011. 